\title{
A highly emissive inorganic hexamolybdenum cluster complex as a handy precursor for the preparation of new luminescent materials
}

Received 00th January 2012, Accepted 00th January 2012

DOI: $10.1039 / \times 0 \times x 00000 x$

\author{
O.A. Efremova, ${ }^{1 a}$ M.A. Shestopalov, ${ }^{1 b}$ N.A. Chirtsova, ${ }^{\text {bc }}$ A.I. Smolentsev, ${ }^{b}$ \\ Y.V. Mironov, ${ }^{b}$ N. Kitamura, ${ }^{d}$ K.A. Brylev ${ }^{* b c}$ and A.J. Sutherland ${ }^{* a}$
}

The synthesis and characterisation of a new, highly luminescent inorganic cluster complex, ()$_{2}\left[{ }_{6}\right]$, are described. The complex possesses labile nitrato ligands and is therefore a useful precursor for the design of new luminescent materials. To exemplify this, functionalised polystyrene beads have been utilised as "polymeric ligands" to immobilise the molybdenum cluster complex.

\section{Introduction}

The superb photoluminescent properties of octahedral molybdenum cluster complexes with the general formula $[\{\}]^{n}$, where $\mathrm{X}$ is an inner ligand $(\mathrm{Cl}, \mathrm{Br}$ or $\mathrm{I})$ and $\mathrm{Y}$ is an outer (apical) organic/inorganic ligand, have led to considerable recent interest in \{\}$^{4+}$-based materials. Such interest stems from the fact that the clusters exhibit photoluminescence in the visible and near-infrared regions with high emission quantum yields and lifetimes of several hundreds of microseconds, and have excellent potential for singlet oxygen generation, ${ }^{1 \mathrm{c},} 2$ etc. Critically, such properties can be tuned readily by facile chemical modification of $\mathrm{X}$ and/or $\mathrm{Y}$ in the complexes. This combination of properties make molybdenum cluster complexes most attractive for a diverse range of practical applications in biology (e.g. for bioimaging and biolabeling ${ }^{3}$ ) and medicine (e.g. as photodynamic therapeutic agents ${ }^{4}$ ). Despite the excellent potential for often insoluble in water and can potentially react in physiological environments. Thus, they must be either coated

\footnotetext{
${ }^{1}$ Equally contributed to the work reported.

a Chemical Engineering and Applied Chemistry, Aston University, Aston Triangle, Birmingham, B4 7ET, UK; Fax: +44 (0)121 204 3679; Tel:+44 (0)121 204 3425; E-mail: a.j.sutherland@aston.ac.uk

${ }^{b}$ Nikolaev Institute of Inorganic Chemistry SB RAS, 3 Acad. Lavrentiev Ave., 630090 Novosibirsk, Russia; Fax: +7(383) 330-94-89; Tel: +7(383) 330-9253; E-mail: kbrylev@gmail.com

${ }^{c}$ Novosibirsk State University, 2 Pirogova Str., 630090 Novosibirsk, Russia

${ }^{d}$ Department of Chemistry, Faculty of Science, Hokkaido University, 0600810 Sapporo, Japan
}

†Electronic Supplementary Information (ESI) available: crystal data (CIF) and supporting results. See DOI: 10.1039/b000000x/

biological use, the known \{\}$^{4+}$-based cluster complexes themselves are not suited for such applications, since they are with appropriate organic ligands or introduced into an inert carrier matrix, e.g. a polymer. Both carrier matrix and ligands must be selected so that they enable the cluster to be tailored for a specific biological application (e.g. addressing delivery; enabling cell membrane permeation, etc.).

Herein we report the synthesis, structure and photophysical properties of photoluminescent octahedral molybdenum cluster compound ( $)_{2}[$ ( )6 (1) and the irreversible immobilisation of this new inorganic cluster into thiol-functionalised polystyrene (PS-SH) microspheres.

\section{Results and Discussion}

\section{Synthesis and characterisation of the cluster complex}

New cluster precursor 1, soluble in organic solvents, was prepared from ( $)_{2}[$ ] by treatment with 6 eq. of using the procedures similar to those reported for the chloride and bromide analogues. According to X-ray single-crystal diffraction analysis, [( $\left.)_{6}\right]^{2-}$ shows the typical structure for octahedral molybdenum cluster complexes (Fig. 1). The molybdenum atoms form an almost perfect octahedron with the Mo-Mo distances in the range 2.6664(9)-2.6776(9) Á. Each triangular face of the octahedron is capped by bridging iodine atoms with the Mo-I distances lying in the range of 2.7667(8)2.7857(8) $\AA$, similar to those of other complexes with the \{\}$^{4+}$ cluster core.', The apical positions around \{\}$^{4+}$ are occupied by nitrato ligands (Mo-O distances are in the range of 2.133(6)-2.154(6) Á). Infrared spectroscopy revealed that the bands at 1488, 1263, and 984

Fig. 1 Structure of the $\left[()_{6}\right]^{2-}$ anion, as observed in $\mathbf{1}$. Thermal ellipsoids are drawn at the $30 \%$ probability level.

$\mathrm{cm}^{-1}$ could be assigned to the ( ), ( ), and $\lambda(\mathrm{NO})$ vibrations, respectively. 


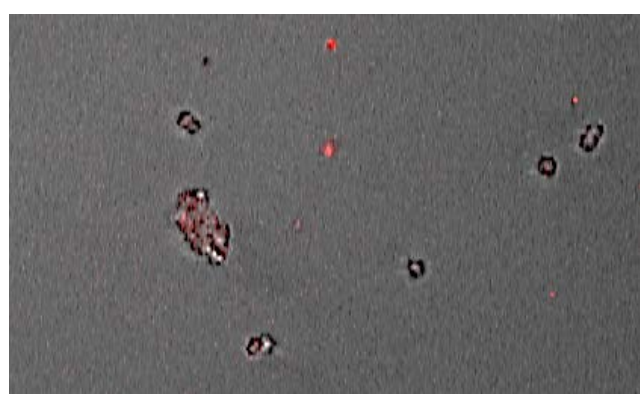

Fig. 3 Confocal microscopy image of \{ \}@PS-SH (2).

dispersion polymerisation reaction ${ }^{9}$ and have been shown to act successfully as "polymeric ligands" for the irreversible immoblisation of quantum dots. ${ }^{10}$ Moreover, PS-based microspheres are ideal materials for certain in vitro biological applications since they are highly biologically inert and recently have been shown to enter a range of cell types via a nonendocytosis-mediated mechanism. ${ }^{11}$ This in turn allows them to serve as excellent vehicles for both binding to a molecular cargo, e.g. a protein or a photoluminescent agent, and delivering it into a cell for delivery/bioimaging purposes.

PS-SH microspheres were obtained via a protocol reported previously $^{10}$ and suspended in a chloroform solution of cluster complex 1. In chloroform, the PS-SH microspheres swelled readily enabling the doping procedure to occur via displacement of the labile nitrato ligands on the cluster complex by the thiol groups present in the polymer matrix. The resultantconjugate material (2) was then washed exhaustively to remove any excess of non-immobilised cluster (UV/vis analysis showed that no cluster was present in the latter washes), treated with a solution of polyvinylpyrrolidinone and resuspended in water giving a stable suspension with a light-yellow colour. This suspension showed bright red luminescence under UV illumination (Fig. S1, ESI $\dagger$ ). Combustion analysis of a dried sample of 2 showed $0.68 \%$ iodine and $0.25 \%$ sulfur corresponding to the \{\}$: S$ ratio of $1: 11$. The XRD results showed that 2 both possesses virtually the same textural properties as pure PS-SH and is free from any crystalline impurities (Fig. S2, ESI $\dagger$ ). The UV/Vis diffuse reflectance spectra displayed an enhanced optical absorption for $\mathbf{2}$ in comparison with pure PS-SH beads due to the presence of molybdenum cluster complex, which is in agreement with the yellow color of the sample (Fig. S3, ESI†). Elemental analysis of the final material 2 showed no evidence of nitrogen in the sample. Similarly, FTIR spectra of $\mathbf{2}$ showed no vibration bands from the - ligands (Fig. S4). Both confirm the successful displacement of all of the nitrato ligands present in 1. Confocal luminescence imaging (Fig. 3) shows clearly that the emission emitted by the molybdenum cluster was indeed localised within the microspheres.

In order to demonstrate that the thiol groups present in the PS-SH are pivotal for binding molybdenum cluster 1, i.e. to prove that the thiol-containing microspheres were indeed acting as "polymeric ligands" a control "blank" experiment was performed (see ESI $\dagger$ for details). Polystyrene (PS) microspheres with no thiol groups were prepared in an identical fashion to the PS-SH microspheres. When these PS microspheres were incubated with a chloroform solution of $\mathbf{1}$, no immobilisation of the cluster complex was evident. These studies indicate that cluster immobilisation occurs solely as aresult of the thiol groups, present in the PS-SH microspheres, acting as ligands for molybdenum cluster $\mathbf{1}$.

To enable the rational development of molybdenum clustercontaining materials it is pivotal to understand the impact that binding of the cluster by the polymer matrix has on the photophysical properties of the cluster units. Our study shows that the powdered sample of conjugate material 2 and its suspension in water display broader emission spectra with $\lambda_{\mathrm{em}}$ being slightly red shifted relative to 1 (Fig. 2, Table 1).

This finding and the decrease in the $\Phi_{\mathrm{em}}$ value for $\mathbf{2}$ in comparison with the starting cluster complex $\mathbf{1}$ is most likely associated with the change of the ligand environment around the \{\} cluster core (i.e. substitution of six - ligands by thiol groups in the polymer matrix). Strikingly, however, the spectroscopic ( $\lambda_{\mathrm{em}}$ and the full-width at half maximum of the emission spectrum $(f w h m))$ and photophysical properties $\left(\Phi_{\mathrm{em}}\right.$ and $\left.\tau_{\mathrm{em}}\right)$ of the suspension of $\mathbf{2}$ in aerated water are almost identical to those of the dry powdered sample of $\mathbf{2}$ (see Table 1). In addition, the emission quantum yield of a suspension of 2 in water (aerated) is almost ten times higher than that of an aerated acetone solution of $\mathbf{1}$. Both of these observations clearly indicate the strong shielding effect that the PS-SH polymer matrix confers against oxygen and solvent quenching of the photoluminescence from the \{\}$^{4+}$ cluster core.

\section{Conclusions}

In conclusion, we have synthesised and characterised ( )2[ ( )6], a new type of octahedral molybdenum cluster complex. This compound displays bright long-lived red phosphorescence in both deaerated solution and the solid phase. Notably, the emission quantum yields of ()$_{2}\left[()_{6}\right]$ are higher than those of any previously reported luminescent metal cluster complex within an exclusively inorganic ligand environment. Complex [ ( )6 $]^{2-}$ bears labile terminal nitrato ligands and consequently is a handy precursor for the preparation of new luminescent materials through substitution of the - groups by an appropriately functionalised polymer matrix. To demonstrate this approach, we have shown that cluster complex $\left[()_{6}\right]^{2-}$ can be readily immobilised into the polymer matrix of PS-SH microspheres (materials used previously for cellular delivery and imaging applications). We have also revealed that the conjugate material shows emission in the red and near-infrared regions arising from metal cluster complex. Such an emission profile is especially promising for bioimaging applications due to relatively low absorption by biological tissues at these wavelengths. Finally, in contrast to the cluster complex [ ( )6 $]^{2-}$, photoluminescence from the conjugate material \{ \}@PS-SH does not show quenching by either solvent or oxygen. This work therefore provides a platform for exploiting these readily 
available and highly luminescent octahedral molybdenum cluster complexes in real applications, such as, bioimaging, cell delivery etc. and it is to this end that we are currently working.

\section{Experimental}

\section{Materials}

Chemical reagents were purchased from Aldrich and used as received, except for styrene which was washed with an aqueous solution of $\mathrm{NaOH}(1 \mathrm{M})$ and dried over magnesium sulphate prior to polymerisation. ( )2[] was synthesised according to earlier reported procedure. ${ }^{12}$ Thiol-containing microspheres were obtained according to the procedure described in. ${ }^{10}$

\section{Equipment}

Elemental analyses were obtained from EuroVector EA3000 Elemental Analyser. FTIR was recorded on Bruker Vertex 80 in $\mathrm{KBr}$ pellet. Energy dispersive X-ray spectroscopy (EDS) was performed on an EDAX equipped (JEOL EX-23000BU) JEOL JSM-6700F field emission scanning electron microscope. Absorption spectra of 1 were recorded in diapason 300-700 nm on a PerkinElmer Lambda35 UV/Vis spectrometer. Confocal microscopy images were obtained on a Zeiss LSM 710 laser scanning confocal microscope with a built on AxioObserver Z1 inverted microscope as the imaging platform. Sample excitation was performed with a 405-nm diode laser. Flow cytometry measurements on \{ \}@PS-SH microspheres suspended in water was performed using a FACSCalibur flow cytometry system. The excitation wavelength was set at $488 \mathrm{~nm}$, while the detection took place at $670 \mathrm{~nm}$. Pure blank PS microspheres were used as controls. X-Ray powder difraction patterns were recorded on a Shimadzu XRD 7000S. Optical diffuse reflectance spectra were measured at room temperature on Shimadzu UV-Vis-NIR 3101 PC spectrophotometer equipped with an integrating sphere and reproduced in the form of Kubelka-Monk theory.

For emission measurements, the powdered samples $\mathbf{1}$ or $\mathbf{2}$ as well as a suspension of $\mathbf{2}$ in water were placed between two nonfluorescent glass plates. The absorbance of an acetone solution of 1 was set $<0.1$ at $355 \mathrm{~nm}$. The solution was poured into two quartz cuvettes and one of them was deaerated by purging with an Ar-gas stream for $30 \mathrm{~min}$ and then the cuvette was sealed. Measurements were carried out at $298 \mathrm{~K}$. The samples were excited by 355-nm laser pulses (6 ns duration, LOTIS TII, LS2137/3). Corrected emission spectra were recorded on a redlight-sensitive multichannel photodetector (Hamamatsu Photonics, PMA-12). For emission decay measurements, the emission was analyzed by a streakscope system (Hamamatsu Photonics, C4334 and C5094). The emission quantum yields (.) for the aerated and deaerated acetone solutions of 1 were estimated by using ( $)_{2}[\mathrm{]}$ as a standard: $=0.19$ in deaerated acetonitrile. ${ }^{13}$ The emission quantum yields of the powdered samples $\mathbf{1}$ and $\mathbf{2}$ along with the water suspension of $\mathbf{2}$ were determined by an Absolute Photo-Luminescence Quantum Yield Measurement System (Hamamatsu Photonics, C9920-03), which comprised an excitation Xenon light source (the excitation wavelength was set at $380 \mathrm{~nm}$ ), an integrating sphere, and a red- sensitive multichannel photodetector (Hamamatsu Photonics, PMA-12).

\section{Synthesis of ( )2 ( )6] (1)}

$500 \mathrm{mg}(0.18 \mathrm{mmol})$ of ( )2[ ] and $200 \mathrm{mg}(1.17 \mathrm{mmol})$ of were dissolved in $20 \mathrm{ml}$ of acetone. The reaction mixture was stirred for $16 \mathrm{~h}$ at room temperature in a flask covered by aluminum foil. The yellow precipitate of AgI was then filtered out. The product was isolated from filtrate by precipitation into excess amount of diethyl ether followed by filtration. Yield: $380 \mathrm{mg}$ (88\%). Anal. calcd. for : C 15.6, H 3.0, N 4.6; found: C 15.9; H 3.1; N 4.5. EDS shows Mo:I = 6:8.1. IR $\left(\mathrm{cm}^{-1}\right): 1488,1263$, 984. UV-vis (acetone): $\quad(\varepsilon)=347 \mathrm{~nm}$ (5950), $394 \mathrm{~nm}$ (5200). The crystals suitable for X-ray diffraction analysis were obtained by evaporation of a hot acetone solution (at $50{ }^{\circ} \mathrm{C}$ ). It was found that 1 crystallises as a solvate with three acetone molecules per formula unit (1·).

\section{Immobilisation of ( $)_{2}\left[()_{6}\right]$ (1) into thiol-containing microspheres, i.e. preparation of \{ \}@PS-SH (2)}

All procedures were carried out at room temperature. Compound 1 (20 mg) was dissolved in (2 $\mathrm{mL})$ and the resultant solution added to an aliquot of PS-SH microspheres (200 mg) suspended in $(2 \mathrm{~mL})$. The reaction mixture was ultrasonicated for 5 minutes and then shaken for $24 \mathrm{~h}$. The solution was mixed with DMF (5 mL) and centrifuged (8 min, $7000 \mathrm{rpm}$ ). The supernatant containing non-immobilised cluster complex was removed by decantation. The separated solid pellet was resuspended in DMF (3 $\mathrm{mL}$ ) by ultrasonication for $\sim 10 \mathrm{~min}$, sedimented by centrifugation ( $8 \mathrm{~min}, 7000 \mathrm{rpm}$ ) and the supernatant removed by decantation. This washing procedure was repeated four times with DMF and twice with methanol. In order to prepare clusterloaded microspheres as a stable suspension in water the clusterloaded microspheres were resuspended in an aqueous solution of PVP ( $=58000 \mathrm{~g} \cdot \mathrm{mol}^{-1}, 1 \% \mathrm{w} / \mathrm{w}, 3 \mathrm{~mL}$ ) by ultrasonication for $\sim 10 \mathrm{~min}$ followed by centrifugation (10 min, $9000 \mathrm{rpm}$ ) and the supernatant removed by decantation. The solid pellet was washed twice with water $(3 \mathrm{~mL})$ by alternate resuspension and centrifugation procedures. Finally, the solid was resuspended in distilled water (2 mL). To obtain a solid sample of \{ \}@PS-SH (2), $100 \mu \mathrm{L}$ of the water suspension was dried to constant mass in a centrifugal evaporator. Anal. found: C 91.5, H 7.54, S 0.25, I 0.68 .

\section{Interaction of non-thiol-containing PS microspheres with cluster complex 1}

The blank (non-thiol-containing) PS microspheres, were generated under identical dispersion polymerisation reaction conditions that were used to produce PS-SH, but in the absence of the functionalised monomer 4-vinylbenzylthiouronium. These blank PS microspheres were treated with the solution of $\mathbf{1}$ and exhaustively washed similarly to that described for $\mathbf{2}$. After that the control sample of PS microspheres was analyzed for molybdenum cluster emission in the red and NIR regions. This analysis, conducted using both confocal microscopy and an optical fibre-based fluorescence probe, showed no emission in 
either region.

\section{Crystal structure determination}

The structure of ( $)_{2}\left[()_{6}\right] \cdot(\mathbf{1} \cdot)$ was solved by single crystal Xray diffraction analysis with the use of a Bruker Nonius X8 Apex 4K CCD diffractometer fitted with graphite monochromatised $\operatorname{MoK}_{\alpha}$ radiation $(\lambda=0.71073 \AA)$. Absorption corrections were made empirically using the SADABS program. ${ }^{14}$ The structures were solved by the direct methods of difference Fourier synthesis and further refined by the full-matrix least-squares method using the SHELXTL program package. ${ }^{14}$ All non-hydrogen atoms were refined anisotropically. The positions of the hydrogen atoms of tetra- $n$-butylammonium cation were calculated corresponding to their geometrical conditions and refined using the riding model. The methyl $\mathrm{C}$ atoms of the $\mathrm{N}$-(n-butyl) 4 chains show apparent signs of disorder over several positions. However, the refinement proceeded with full weighted atoms because an adequate disorder model could not be resolved using the room-temperature diffraction data collected. The structure contains a number of solvent acetone molecules, which appeared to be highly disordered and it was difficult to model their positions reliably. Therefore, the structure was treated via the PLATON/SQUEEZE ${ }^{15}$ procedure to remove the contribution of the electron density in the solvent regions from the intensity data, and the solvent-free model was employed from the final refinement. PLATON calculations indicated that the effective volume for solvent molecules is $582 \AA^{3}$, which were assigned to 6 acetone molecules per unit cell or 3 acetone molecules per formula unit. Crystal data for : $\mathrm{M}=2622.05$, monoclinic, $/ n, a=$ 14.5949(4) $\AA, b=11.4521(3) \AA, c=22.3086(5) \AA, \beta=$ 95.8350(10) ${ }^{\circ}, \mathrm{Z}=2, \mathrm{~V}=3709.39(16) \AA^{3}, \quad=2.348 \mathrm{~g} * \mathrm{~cm}-3, \mathrm{~T}=$ 296(2) K, $=0.0339,=0.0469,=0.1463, S=0.936$. CCDC 949910 contains the supplementary crystallographic data for this paper. These data can be obtained free of charge from the Cambridge Crystallographic Data Centre (.ccdc.cam.ac.uk/data_request/cif).

\section{Acknowledgements}

This research was supported by a Marie Curie Intra-European Fellowship (project "PolyMoRe", No. 327440) and by the Russian Foundation for Basic Research (Grant No. 12-0331670). Also K. A. Brylev thanks the Japan Society for the Promotion of Science (JSPS) for a Post Doctoral Fellowship for Foreign Researchers.

\section{Notes and references}

1. (a) A. W. Maverick, J. S. Najdzionek, D. Mackenzie, D. G. Nocera and H. B. Gray, J. Am. Chem. Soc., 1983, 105, 1878-1882; (b) M. N. Sokolov, M. A. Mihailov, E. V. Peresypkina, K. A. Brylev, N. Kitamura and V. P. Fedin, Dalton Trans., 2011, 40, 6375-6377; (c) K. Kirakci, P. Kubat, M. Dusek, K. Fejfarova, V. Sicha, J. Mosinger and K. Lang, Eur. J. Inorg. Chem., 2012, 3107-3111; (d) K. Kirakci, P. Kubat, J. Langmaier, T. Polivka, M. Fuciman, K. Fejfarova and K. Lang, Dalton Trans., 2013, 42, 7224-7232.

2. J. A. Jackson, M. D. Newsham, C. Worsham and D. G. Nocera, Chem. Mater., 1996, 8, 558-564.
3. (a) S. Cordier, F. Dorson, F. Grasset, Y. Molard, B. Fabre, H. Haneda, T. Sasaki, M. Mortier, S. Ababou-Girard and C. Perrin, J. Cluster Sci., 2009, 20, 9-21; (b) F. Grasset, F. Dorson, Y. Molard, S. Cordier, V. Demange, C. Perrin, V. Marchi-Artzner and H. Haneda, Chem. Commun., 2008, 4729-4731; (c) F. Grasset, F. Dorson, S. Cordier, Y. Molard, C. Perrin, A. M. Marie, T. Sasaki, H. Haneda, Y. Bando and M. Mortier, Adv. Mater., 2008, 20, 143-148.

4. D. E. J. G. J. Dolmans, D. Fukumura and R. K. Jain, Nat. Rev. Cancer, 2003, 3, 380-387.

5. P. Braack, M. K. Simsek and W. Preetz, Z. Anorg. Allg. Chem., 1998, 624, 375-380.

6. P. Bruckner, W. Preetz and M. Punjer, Z. Anorg. Allg. Chem., 1997, 623, 8-17.

7. J. A. Jackson, C. Turro, M. D. Newsham and D. G. Nocera, J. Phys. Chem., 1990, 94, 4500-4507.

8. (a) L. F. Szczepura, K. A. Ketcham, B. A. Ooro, J. A. Edwards, J. N. Templeton, D. L. Cedeno and A. J. Jircitano, Inorg. Chem., 2008, 47, 7271-7278; (b) L. F. Szczepura, J. A. Edwards and D. L. Cedeno, J. Cluster Sci., 2009, 20, 105-112.

9. S. Kawaguchi and K. Ito, Polymer Particles, 2005, 175, 299-328.

10. J. M. Behrendt, M. Afzaal, L. M. Alexander, M. Bradley, A. V. Hine, D. Nagel, P. O'Brien, K. Presland and A. J. Sutherland, J. Mater. Chem., 2009, 19, 215-221.

11. (a) L. M. Alexander, S. Pernagallo, A. Livigni, R. M. SanchezMartin, J. M. Brickman and M. Bradley, Mol. BioSyst., 2010, 6, 399409; (b) J. M. Behrendt, D. Nagel, E. Chundoo, L. M. Alexander, D. Dupin, A. V. Hine, M. Bradley and A. J. Sutherland, Plos One, 2013, 8, e50713.

12. P. Bruckner, W. Preetz and M. Punjer, Z. Anorg. Allg. Chem., 1997, 623, 8-17.

13. A. W. Maverick, J. S. Najdzionek, D. Mackenzie, D. G. Nocera and H. B. Gray, J. Am. Chem. Soc., 1983, 105, 1878-1882.

14 Bruker (2004), APEX2 (Version 1.08), SAINT (Version 7.03), SADABS (Version 2.11), SHELXTL (Version 6.12), Bruker AXS Inc., Madison, WI, USA

15 (a) A. L. Spek, J. Appl. Cryst., 2003, 36, 7-13; (b) P. Vandersluis and A. L. Spek, Acta Crystallogr., Sect. A: Found. Crystallogr., 1990, 46, 194-201. 